\title{
HIV in men who have sex with men in Lebanon: clinical \& psychosocial aspects
}

Ismael Maatouk, MD, MPH${ }^{1}$, Rusi Jaspal, $\mathrm{PhD}^{2}$.

${ }^{1}$ Dermatology department, Clemenceau Medical Center affiliated with Johns Hopkins (ismaelmaatouk@gmail.com);

Faculty of Health \& Life Sciences, De Montfort University, Leicester LE1-9BH, UK (ismael.maatouk@dmu.ac.uk)

${ }^{2}$ Faculty of Health \& Life Sciences, De Montfort University, Leicester LE1-9BH, UK (rusi.jaspal@cantab.net)

Running title: HIV and MSM in Lebanon

Word count of text: 1070

Number of figures: 0

Corresponding author

Ismael Maatouk, MD, MPH

${ }^{1}$ Dermatology department, Clemenceau Medical Center affiliated with Johns Hopkins (ismaelmaatouk@gmail.com);

Faculty of Health \& Life Sciences, De Montfort University, Leicester LE1-9BH, UK

(ismael.maatouk@dmu.ac.uk)

Tel: +9613568968

Keywords

HIV; men who have sex with men; Lebanon; fast-track; sexually transmitted infections

Conflicts of interest

None declared for IM and RJ

Citing this article

Maatouk, I., \& Jaspal, R. (in press). HIV in men who have sex with men in Lebanon: Clinical \& psychosocial aspects. BMJ Sexual \& Reproductive Health. 


\section{HIV in men who have sex with men in Lebanon: clinical \& psychosocial aspects}

A concerted global effort to end HIV transmissions is necessary. In the Middle East and North Africa (MENA), men who have sex with men (MSM) constitute a hard-to-reach population, due to the social stigma and criminalisation of homosexuality in most countries.[1] When compared to other MENA countries, Lebanon is one of the safest for sexual minority groups. Its capital city, Beirut, is one of the most socially progressive cities in MENA, with a vibrant MSM community and gay bars, clubs and community centers.[2] Yet, we know little about HIV prevalence, incidence and risk factors in MSM in Lebanon, a group disproportionately affected by the condition. This editorial sets an agenda for HIV research in this key population.

HIV care in Lebanon is offered through a collaboration between the National AIDS Control Program (NACP) (jointly funded the Lebanese state and the World Health Organization) and various non-governmental organisations (NGOs). The NACP provides the NGOs with training, and provides access to HIV testing, and antiretroviral therapy for Lebanese citizens and refugees. Sexual health care is available through gay-friendly NGOs which are widely known in the Lebanese gay community as a space for accessing low-cost STI testing and sexual healthcare. The cost of comprehensive care, clinical monitoring of HIV patients, and the treatment of other health issues (e.g. mental health) are not covered by the NACP, which can undermine the quality of HIV care. The NACP also provides access to post-exposure prophylaxis (PEP) but not to pre-exposure prophylaxis (PrEP), which must be acquired privately. The exclusion of sexual healthcare from primary healthcare settings, the absence of sexual health coverage by private insurance companies, the instability of the Lebanese healthcare system, and HIV stigma, collectively, inhibit consistent access to HIV care.

According to the NACP, the HIV epidemic in Lebanon has remained at a plateau for the past 10 years, with relatively few recorded HIV-related deaths.[3] Until December 2017, 2206 HIV/AIDS cases were recorded.[3] The epidemic is concentrated in MSM and incidence has increased from at least 47.7\% MSM of all HIV diagnoses in 2016 to at least $54.15 \%$ in 2017 (38.3\% of the reported cases in 2016 had a "not specified" sexual orientation versus $9.8 \%$ in 2017).[3] According to a data synthesis, 3500 people were estimated to be living with HIV in Lebanon in 2011 (less than $0.01 \%$ of the general population and $3.7 \%$ of MSM).[2] However, these data are now at least 7 years old. In a study of 213 MSM, a prevalence of $1.5 \%$ was reported (3/197),[2] while another study in 2017, using a similar sampling strategy, reported a prevalence of $12.3 \%$ in 292 MSM, $25 \%$ of whom were Syrian refugees. [5] In short, we do not yet have reliable data on HIV epidemiology in MSM in Lebanon and other potential secondary makers of HIV infection, such as syphilis and gonorrhea prevalence, are not reported to the NACP.

The lack of data can be attributed partly to low rates of HIV testing. According to a 2012 UNAIDS report, testing rates in the MENA region are among the lowest in the world.[6] Testing rates among Lebanese MSM ranged from 25\% in 2010 to 38\% in 2014. $[2,4]$ However, these data may not be representative of MSM in Lebanon for at least two reasons. First, studies have generally included more MSM in relationships, who are much more likely to have tested for HIV than men not in relationships, potentially causing selection bias.[2] Second, participant samples have included a large number of MSM engaged in sex work (36\% according to one study). [4] Given the nature of their work, MSM sex workers report having a higher number of sexual partners (2-3 partners/day) than MSM who do not sell sex (10 partners/year).[4] This group is less likely to use condoms and to access health services, due to socio-structural barriers such as poverty, stigma, legal issues. Furthermore, Wagner et al. reported a testing rate of $62 \%$ in their study of 213 MSM from 
Beirut [2]. Although the overall testing rates appear to have increased, there is a need to increase its prevalence and frequency in MSM.

Existing data indicate a high prevalence of sexual risk-taking in Lebanese MSM.[2,4,5] In a study of 292 MSM, it was found that $65 \%$ reported condomless anal sex (CAS) and that $22 \%$ disclosed engaging in group sex.[5] In another study of $213 \mathrm{MSM}, 23 \%$ of participants reported CAS with a partner living with HIV or of unknown status.[2] Current research suggests that condom use is determined by relationship context and type,[2] and one study has shown that men in 'committed relationships' were almost 3 times more likely to engage in CAS when the HIV status of their sex partner was known.[7] A study of MSM sex workers has shown a high prevalence of CAS with regular partners and a high prevalence of inconsistent condom use with clients.[7] However, more data are needed to understand the psychosocial correlates of risk-taking behavior.

Data on HIV care are also limited. In 2017, the cumulative number of people living with HIV in receipt of HIV care from the NACP reached $1320(1320 / 2206=59.83 \%)$.[3] Treatment coverage and the rate of viral suppression in HIV-positive MSM are not known. There is some work on wellbeing among Lebanese HIV patients, with one study reporting a fair quality of life in patients.[8] Symptomatic HIV and perceived stigma were negative predictors of quality of life. HIV status disclosure is also known to predict wellbeing. Although $80.5 \%$ of participants reported disclosing their HIV status to someone else, $72.5 \%$ reported being cautious about disclosure to avoid stigma and marginalization. [8] Understanding engagement with care, quality of life and HIV status disclosure in Lebanese MSM living with HIV is vital.

This editorial illustrates an urgent need for more reliable data on HIV prevalence, incidence and risk factors associated in Lebanese MSM, as well as the rate and nature of patient engagement with HIV care. The studies described enable us to identify the key characteristics of the HIV epidemic in Lebanon. Yet, the data also show that rates of HIV testing are unacceptably low. Behavioural interventions, higher rates of HIV testing and engagement with HIV care depend on our ability to understand medical, behavioural, and cultural drivers. Future studies must recruit representative samples of MSM and focus on the psychosocial correlates of risk-taking. However, systematic research into HIV remains a challenge in Lebanon where social, cultural and religious norms around sexuality contribute to the stigmatisation and exclusion of MSM.

\section{References}

[1] Gökengin D, Doroudi F, Tohme J et al..HIV/AIDS: trends in the Middle East and North Africa region. Int J Infect Dis 2016;44:66-73.

[2] Wagner GJ, Hoover M, Green H et al. Social, Relational and Network Determinants of Unprotected Anal Sex and HIV Testing Among Men Who Have Sex with Men in

Beirut, Lebanon. Int J Sex Health 2015;27(3):264-75.

[3] National Aids Control Program, 2017 statistics, Lebanon

[4] Mumtaz G, Hilmi N, McFarland W et al. Are HIV Epidemics among Men Who Have Sex with Men Emerging in the Middle East and North Africa?: A Systematic Review and Data Synthesis. PLoS Medicine 2011;8(8):e1000444

[5] Heimer R, Barbour R, Khouri D et al. HIV risk, prevalence and access to care among men who have sex with men in Lebanon. AIDS Res Hum retroviruses 2017;33:11.

[6] UNAIDS. 2012 Country Progress Report for Lebanon. 2013.

[7] Aunon FM, Wagner GJ, Maher R et al. An exploratory study of HIV risk behaviours and testing among male sex workers in Beirut, Lebanon. Soc Work Public Health

2015;30(4):373-384. 
[8] Abboud S, Noureddine S, Abu-Saad Huijer H et al. Quality of life in people living with HIV/AIDS in Lebanon. AIDS Care 2010; 22:6, 687-96 